\title{
AUTHOR INDEX \\ VOLUME 12 (1998)
}

Alford, C.O., see Kubota, T.

$12(1998) 335-354$

Ancona, F., Rovetta, S. \& Zunino, R., An image-recognition system implemented on hierarchical parallel architectures

Barlos, F., see Bourbakis, N.G.

Bensaid, A.M. \& Bezdek, J.C., Semi-supervised point prototype clustering

Berka, P. \& Bruha, I., Empirical comparison of various discretization procedures

Betrouni, M., see Hamad, D.

Bezdek, J.C., see Bensaid, A.M.

Bianchi, N., Bottoni, P., Mussio, P., Spinu, C. \& Garbay, C., Situated image understanding in a multiagent framework

Biela, P., see Hamad, D.

Blostein, D., see Fahmy, H.

Bottoni, P., see Bianchi, N.

Bouchon-Meunier, B., see Gascuel, O.

Boufama, B.S. \& Mohr, R., A stable and accurate algorithm for computing epipolar geometry

Bourbakis, N., see Moghaddamazadeh, A.

Bourbakis, N.G., Preface

Bourbakis, N.G., Barlos, F. \& Mertoguno, J.S., HERMES autonomous vision system: the flat quadtree model

Bowyer, K., Stark, L. \& Stockman, G., Preface

Bruha, I., see Berka, P.

Bunke, H., see Messmer, B.T.

Caelli, T., see Lazarescu, M.M.

Caraux, G., see Gascuel, O.

Chau, C.W., see Kwong, S.

Chaudhuri, B., see Sengupta, P.

Chen, J.-M., see Lee, H.-M.

Cheng, H.D. \& Desai, R., Scene classification by fuzzy local moments

Cho, H.-G., see Lee, D.-H.

Chung, K., see Seo, K.

Crisman, J.D., see Lopresti, D.P.

Dai, R., see Xiao, X.

Desai, R., see Cheng, H.D.

Di Ruberto, C., see Vitulano, S.

Ding, J., see Lam, L.

Drets, G. \& Liljenström, H., Fingerprint sub-classification and singular point detection

Dudek-Dyduch, E., see Dyduch, T.

Dyduch, T. \& Dudek-Dyduch, E., Application of neural networks in $3 \mathrm{D}$ object recognition system

12 (1998) 293-310

12 (1998) 265-291

12 (1998) 625-643

12 (1998) 1017-1032

12 (1998) $505-516$

12 (1998) 625-643

12 (1998) 595-624

12 (1998) 505-516

12 (1998) 763-799

12 (1998) $595-624$

12 (1998) 517-571

12 (1998) 817-840

12 (1998) 801-816

12 (1998) 263-264

12 (1998) 265-291

12 (1998) 1033-1034

12 (1998) 1017-1032

12 (1998) 721-742

12 (1998) 867-879

12 (1998) 517-571

12 (1998) 573-594

12 (1998) 695-720

12 (1998) 743-761

12 (1998) 921-938

12 (1998) 45-61

12 (1998) 251-261

12 (1998) 119-141

12 (1998) 143-157

$12(1998) 921-938$

12 (1998) $677-694$

12 (1998) 63-79

12 (1998) 407-422

12 (1998) 491-504

12 (1998) 491-504 
Ewert, S. \& van der Walt, A., Generating pictures using random forbidding context

Fahmy, H. \& Blostein, D., A graph-rewriting paradigm for discrete relaxation: application to sheet-music recognition

Feng, G.C., see Yuen, P.C.

Fisher, R. B. \& Koryllos, K., Interactive textbooks; embedding image processing operator demonstrations in text

12 (1998) 209-221

12 (1998) 1095-1123

Foresti, G.L., A line segment based approach for 3D motion estimation and tracking of multiple objects

Fuh, C.-S., see Kao, S.-L.

Gallinari, P., see Gascuel, O.

Garbay, C., see Bianchi, N.

Gascuel, O., Bouchon-Meunier, B., Caraux, G., Gallinari, P., Guénoche, A., Guermeur, Y., Lechevallier, Y., Marsala, C., Miclet, L., Nicolas, J., Nock, R., Ramdani, M., Sebag, M., Tallur, B., Venturini, G. \& Vitte, P., Twelve numerical, symbolic and hybrid supervised classification methods

Goldgof, D., see Sarkar, S.

Goldman, D., see Moghaddamazadeh, A.

Guénoche, A., see Gascuel, $\mathrm{O}$.

Guerfali, W., see Plamondon, R.

Guermeur, Y., see Gascuel, O.

Hamad, D., Betrouni, M., Biela, P. \& Postaire, J.-G., Neural networks inspection system for glass bottles production: a comparative study

Han, T.-D., see Kim, J.-M.

He, Q.H., see Kwong, S.

Heikkonen, J. \& Herpers, R., Editorial

Heikkonen, J. \& Varfis, A., Land cover/land use classification of urban areas: a remote sensing approach

12 (1998) 881-900

12 (1998) 951-968

12 (1998) $517-571$

12 (1998) 595-624

12 (1998) 517-571

12 (1998) 1071-1080

12 (1998) 801-816

12 (1998) 517-571

12 (1998) 31-44

12 (1998) 517-571

12 (1998) 505-516

12 (1998) 311-334

12 (1998) $573-594$

12 (1998) 379-380

Herpers, R. \& Sommer, G., Discrimination of facial regions based on dynamic grids of point representations

$12(1998) 475-489$

12 (1998) 381-405

12 (1998) 379-380

Hong, C., Loudon, G., Wu, Y. \& Zitserman, R., Segmentation and recognition of continuous handwriting Chinese text

12 (1998) 223-232

12 (1998) 985-1005

Huang, Y.S., Liu, K., Suen, C.Y. \& Tang, Y.Y., A reliability design methodology for Chinese character recognition

Huntsberger, T., see Kubota, T.

Iñesta, J.M., see Sanchiz, J.M.

Kao, S.-L. \& Fuh, C.-S., Near point light sources for shape from shading

Kim, J., see Seo, K.

Kim, J.H., see Sin, B.-K.

Kim, J.-M., Kim, Y., Kim, S.-D., Han, T.-D. \& Yang, S.-B., An adaptive parallel computer vision system

12 (1998) $159-172$

12 (1998) 335-354

12 (1998) 661-675

12 (1998) 951-968

12 (1998) 251-261

12 (1998) 233-249

12 (1998) 311-334

12 (1998) 311-334

Kim, S.-D., see Kim, J.-M.

12 (1998) 81-95

12 (1998) 311-334

12 (1998) 1095-1123

Koryllos, K., see Fisher, R. B. 
Kubota, T., Huntsberger, T. \& Alford, C.O., A vision system with real-time feature extractor and relaxation network

Kuo, C.-C., see Tseng, Y.-H.

Kwong, S., He, Q.H., Man, K.F., Tang, K.S. \& Chau, C.W., Parallel genetic-based hybrid pattern matching algorithm for isolated word recognition

Laaksonen, J. \& Oja, E., Learning subspace classifiers and error-corrective feature extraction

Lam, L., Ding, J. \& Suen, C.Y., Differentiating between Oriental and European scripts by statistical features

Landraud-Lamole, A.M., Principle of a parallel vision system adapted to textures. A theoretical solution for selecting visual filters

Lazarescu, M.M., Caelli, T. \& Venkatesh, S., Extracting common subtrees from decision trees

Lechevallier, Y., see Gascuel, $\mathrm{O}$.

Lee, D.-H. \& Cho, H.-G., A new synthesizing method for handwriting Korean scripts

Lee, H.-J., see Tseng, Y.-H.

Lee, H.-M., Lin, C.-C. \& Chen, J.-M., A preclassification method for handwritten Chinese character recognition via fuzzy rules and SEART neural net

Lee, S.-W., see Kim, S.-Y.

Lee, S.-W., see Park, H.-S.

Lee, S.W., Tang, Y.Y. \& Wang, P.S.P., Preface

Li, X., see Plamondon, R.

Liljenström, H., see Drets, G.

Lin, C.-C., see Lee, H.-M.

Lin, C.-F. \& Hsiao, C.-Y., Structural recognition for table-form documents using relaxation techniques

Liu, J. \& Tang, Y.Y., Distributed autonomous agents for Chinese document image segmentation

Liu, K., see Huang, Y.S.

Lopresti, D.P., Ma, M.Y., Wang, P.S.P. \& Crisman, J.D., Ink matching of cursive Chinese handwritten annotations

Loudon, G., see Hong, C.

Ma, M.Y., see Lopresti, D.P.

Man, K.F., see Kwong, S.

Marsala, C., see Gascuel, O.

Maxwell, B. A., Teaching computer vision to computer scientists: issues and a comparative textbook review

Mertoguno, J.S., see Bourbakis, N.G.

Messmer, B.T. \& Bunke, H., Error-correcting graph isomorphism using decision trees

12 (1998) 335-354

12 (1998) 173-190

12 (1998) 573-594

12 (1998) 423-436

12 (1998) 63-79

12 (1998) 355-378

12 (1998) 867-879

12 (1998) 517-571

$12(1998) 45-61$

12 (1998) 173-190

12 (1998) 743-761

12 (1998) 81-95

12 (1998) 191-208

12 (1998) 1-4

12 (1998) 31-44

12 (1998) 407-422

12 (1998) 743-761

12 (1998) 985-1005

12 (1998) 97-118

12 (1998) 159-172

12 (1998) 119-141

12 (1998) 223-232

12 (1998) 119-141

12 (1998) 573-594

12 (1998) 517-571

12 (1998) 1035-1051

12 (1998) 265-291

12 (1998) 721-742

12 (1998) 517-571

Miclet, L., see Gascuel, O.

Moghaddamazadeh, A., Goldman, D. \& Bourbakis, N., A fuzzy-like approach for smoothing and edge detection in color images

12 (1998) 801-816

Mohanty, S., Pattern recognition in alphabets of Oriya language using Kohonen neural network

Mohr, R., see Boufama, B.S.

12 (1998) 1007-1015

12 (1998) 817-840

Mori, S., see Suen, C.Y.

12 (1998) 5-29 
Murphy, R. R., Teaching image computation in an upper level elective on robotics

Mussio, P., see Bianchi, N.

Nappi, M., see Vitulano, S.

Ng, G.S. \& Sim, H.C., Recognition of partially occluded objects with back-propagation neural network

Nicolas, J., see Gascuel, O.

Nock, R., see Gascuel, O.

Oja, E., see Laaksonen, J.

Oja, E., see Valkealahti, K.

Ortega, A. \& Shah, M., From shape from shading to object recognition

Park, H.-S., Song, H.-H. \& Lee, S.-W., A self-organizing hierarchical classifier for multi-lingual large-set Oriental character recognition

Petrou, M., see Stassopoulou, A.

Pla, F., see Sanchiz, J.M.

Plamondon, R., Guerfali, W. \& Li, X., The generation of Oriental characters characters: new perspectives for automatic handwriting processing

Postaire, J.-G., see Hamad, D.

Ramdani, M., see Gascuel, O.

Rim, H.-C., see Suen, C.Y.

Rosin, P.L., Refining region estimates

Rovetta, S., see Ancona, F.

Sanchiz, J.M., Pla, F. \& Iñesta, J.M., Using neural networks to detect dominant points in chain-coded contours

12 (1998) 1081-1093

12 (1998) 595-624

12 (1998) 677-694

12 (1998) 645-660

$12(1998) 517-571$

12 (1998) 517-571

12 (1998) 423-436

12 (1998) 437-452

12 (1998) $969 \quad 984$

12 (1998) 191-208

12 (1998) $901-920$

12 (1998) 661-675

Sarkar, S. \& Goldgof, D., Integrating image computation in undergraduate level data-structure education

Sebag, M., see Gascuel, O.

Sengupta, P. \& Chaudhuri, B., A "generalized" lexical functional grammar-based processing of an Indian language - Bangla

Seo, K., Kim, J., Yoon, J. \& Chung, K., Comparison of feature performance and its application to feature combination in off-line handwritten Korean alphabet recognition

Shah, M., see Ortega, A.

Sim, H.C., see $\mathrm{Ng}$, G.S.

Sin, B.-K. \& Kim, J.H., Network-based approach to Korean handwriting analysis

Sommer, G., see Herpers, R.

Song, H.-H., see Park, H.-S.

Spinu, C., see Bianchi, N.

Stark, L., see Bowyer, K.

Stassopoulou, A. \& Petrou, M., Obtaining the correspondence between Bayesian and neural networks

Stockman, G., see Bowyer, K.

Suen, C.Y., see Huang, Y.S.

Suen, C.Y., see Lam, L.

Suen, C.Y., Mori, S., Rim, H.-C. \& Wang, P.S.P., Intriguing aspects of Oriental languages

12 (1998) 31-44

12 (1998) 505-516

12 (1998) $517-571$

12 (1998) 5-29

12 (1998) $841-866$

12 (1998) 293-310

12 (1998) 661-675

12 (1998) 1071-1080

12 (1998) 517-571

12 (1998) 695-720

12 (1998) 251-261

12 (1998) 969-984

12 (1998) 645-660

12 (1998) 233-249

12 (1998) 381-405

12 (1998) 191-208

12 (1998) 595-624

12 (1998) 1033-1034

12 (1998) 901-920

12 (1998) 1033-1034

12 (1998) 159-172

12 (1998) 63-79

$12(1998) 5-29$

12 (1998) $517-571$

Tallur, B., see Gascuel, O. 
Tang, K.S., see Kwong, S.

$12(1998) 573-594$

Tang, Y.Y, see Lee, S.W.

$12(1998) 1-4$

Tang, Y.Y., see Huang, Y.S.

12 (1998) $159-172$

Tang, Y.Y., see Lin, J.

12 (1998) $97-118$

Tang, Y.Y., see Yuen, P.C.

12 (1998) 209-221

Tanimoto, S. L., Connecting middle school mathematics to computer vision and pattern recognition

Tseng, Y.-H., Kuo, C.-C. \& Lee, H.-J., Typeface identification for printed Chinese characters

Utschick, W., A regularization method for non-trivial codes in polychotomous classification

Valkealahti, K. \& Oja, E., Texture classification with singleand multiresolution co-occurrence maps

van der Walt, A, see Ewert, S.

Varfis, A., see Heikkonen, J.

Venkatesh, S., see Lazarescu, M.M.

Venturini, G., see Gascuel, $\mathrm{O}$.

Vitte, P., see Gascuel: O.

Vitulano, S., Di Ruberto, C. \& Nappi, M., Edge detection: local

$12(1998) 1053-1070$

$12(1998) 173-190$

$12(1998) 453-474$

$12(1998) 43 i-452$

$12(1998) 939-950$

12 (1998) $475-489$

$12(1998) 867-879$

12 (1998) $517-571$

$12(1998) 517-571$ and global operators

Wang, P.S.P., see Lee, S.W.

Wang, P.S.P., see Lopresti, D.P.

Wang, P.S.P., see Suen, C.Y.

Wu, Y., see Hong, $\mathrm{C}$.

Xiao, X. \& Dai, R., On-line handwritten Chinese character recognition directed by components with dynamic templates

Yang, S.-B., see Kim, J.-M.

Yoon, J., see Seo, K.

Yuen, P.C., Feng, G.C. \& Tang, Y.Y., Printed Chinese character similarity measurement using ring projection and distance transform

Zitserman, R., see Hong, C.

Zunino, R., see Ancona, F.

$12(1998) 677-694$

12 (1998) 1-4

12 (1998) 119-141

12 (1998) 5-29

$12(1998) 223-232$

12 (1998) $143-157$

12 (1998) 311-334

12 (1998) 251-261

12 (1998) 209-221

12 (1998) 223-232

12 (1998) 293-310 